\title{
On antiresonance in the forced response of mistuned bladed disks
}

\author{
Keith Jones and Charles Cross \\ Propulsion Directorate, Air Force Research Laboratory, 1950 Fifth Street, Wright Patterson AFB, OH 45433-7251, \\ USA
}

Received 14 January 2002

Revised 2 September 2002

\begin{abstract}
Mistuning in bladed disks usually increases the forced response of the maximum responding blade leading to shortened component life in turbine engines. This paper investigates mistuning using a transfer function approach where the frequency response functions (FRFs) are described by natural frequencies and antiresonant frequencies. Using this approach, antiresonant frequencies are shown to be a critical factor in determining the maximum blade response. Two insights are gained by formulating antiresonant frequencies as the eigenvalues of reduced system matrices: 1) Mistuning a particular blade has no effect on that blade's antiresonant frequencies. 2) Engine orders $\mathrm{N}$ and $\mathrm{N} / 2$, where $\mathrm{N}$ is the number of blades on the disk, tend to produce the highest maximum local response. Numerical examples are given using a spring-mass-oscillator model of a bladed disk. Pole-zero loci of mistuned bladed disks show that increased maximum blade response is often due to the damping of antiresonant frequencies. An important conclusion is that antiresonant frequencies can be arranged such that a mistuned bladed disk has a lower maximum blade response than a tuned bladed disk.
\end{abstract}

\section{Nomenclature}

A Mistuning amplification factor

$B \quad$ Input shape matrix

C Output shape matrix

E Engine order

$F \quad$ Vector of applied forces

$F R F \quad$ Frequency response function $(20 \log |h|)$

$G \quad$ Transfer function gain

$h \quad$ Blade frequency response

I Identity matrix

$i \quad$ Blade number

j $\quad \sqrt{-1}$

$K \quad$ Global stiffness matrix

$k_{b} \quad$ Blade stiffness

$k_{c} \quad$ Coupling stiffness

$M \quad$ Global mass matrix

$m \quad$ Nominal blade mass

$m_{i} \quad$ Individual blade mass

$N \quad$ Number of blades

$p \quad$ System poles (natural frequencies)

$p_{u} \quad$ Undamped poles

$R \quad$ Coupling ratio

$\begin{array}{ll}x & \text { Displacement degree of freedom } \\ y & \text { Measured output } \\ z & \text { Transfer function zeros (antiresonant } \\ & \text { frequencies) } \\ z_{u} & \text { Undamped zeros } \\ u & \text { Harmonic input excitation } \\ \phi_{i n} & \text { The } i^{t h} \text { element in the } n^{t h} \text { mode shape } \\ \phi_{n} & \text { The } n^{t h} \text { mode shape } \\ \omega & \text { Excitation frequency } \\ \omega_{b} & \text { Nominal blade natural frequency } \\ \theta & \text { Interblade phase angle } \\ \zeta & \text { Damping factor }\end{array}$

\section{Background}

\subsection{Mistuning}

As bladed disks rotate in turbine engines, the airfoils experience alternating forces resulting from disturbances in the flow field. This excitation is often 
called engine order excitation where the engine order refers to the number of equally spaced disturbances due to struts, vanes, or stators either upstream or downstream of the bladed disk. The resulting blade vibration causes fatigue cracks to initiate and grow. This process is called high cycle fatigue ( $\mathrm{HCF}$ ) because of the tremendous number of cycles accumulated at the high rotational rates of turbine engines. (HCF can also be present in stationary airfoils due to rotating pressure disturbances.) The U.S. Air Force spends a significant amount of money and effort inspecting and fixing $\mathrm{HCF}$ problems.

HCF is exacerbated by mistuning, a breakdown in the cyclic periodicity of bladed disks. A tuned bladed disk is a perfectly cyclic symmetric structure where all blades are identical. Unfortunately, manufacturing tolerances will inevitably cause small differences in the geometries, material properties, or interface boundary conditions of blades resulting in what is called a mistuned bladed disk. The maximum resonant response of any blade on the disk, or maximum response for short, is usually greater for mistuned bladed disk than for a tuned bladed disk [1]. Therefore, mistuning shortens component life, and negatively impacts the durability and reliability of engines.

Most of the mistuning literature has relied on a modal approach when investigating bladed disk forced response. The modal approach relates natural frequency and mode shape behavior to forced response using concepts such as eigenvalue veering and mode localization. Examples of the modal approach include papers by Ewins [2], Wei and Pierre [3,4], and Brewer et al. [5] After surveying the mistuning literature, Slater et al. [6] concluded that amplified maximum response in mistuned bladed disks is due to light damping and mode localization.

\subsection{Antiresonance}

This paper investigates mistuning using a transfer function approach where the forced response is formulated in terms of natural frequencies and antiresonant frequencies. This approach avoids using mode shapes, which become disordered in the presence of mistuning [3] making their effect on maximum response difficult to understand. Instead, the antiresonant frequencies incorporate the mode shape information while providing a more direct and intuitive link to maximum forced response levels.

Antiresonance is an area of continuing research in the structural dynamics community. Miu [7] showed that for discrete spring-mass systems antiresonant frequencies are the resonant frequencies of some substructure. These substructures and their constraints are defined by La Civita [8] in continuous one dimensional systems. Wahl and Schmidt [9] applied these discoveries to experimental structural analysis. Jones and Turcotte [10], D'Ambrogio and Frengolent [11], Rade et al. [12], and Lallement and Cogan [13] have applied antiresonance to finite element model updating with promising results.

These papers analyzed antiresonance in single-input single-output (SISO) transfer functions. Bladed disk forced response requires a multi-input single-output (MISO) method, because engine order excitation is applied at all blades. Wang's [14] formulation of antiresonance can accommodate MISO problems and is applied to models of bladed disks in this paper.

Although the transfer function forced response formulation is mathematically equivalent to the modal formulation, it does provide a different perspective on mistuning phenomena. The insights that come from understanding antiresonance may provide new approaches for reducing maximum response in bladed disks.

\section{Theory}

\subsection{Forced response formulation}

The undamped equations of motion (EOM) for a bladed disk assembly can be written as:

$$
\begin{aligned}
& M \ddot{x}+K x=F=B u \\
& y=C x
\end{aligned}
$$

$$
\begin{aligned}
M & =\left[\begin{array}{ccccc}
m_{11} & m_{12} & 0 & \cdots & m_{21} \\
m_{21} & m_{22} & m_{12} & 0 & \cdots \\
\cdots & \cdots & \cdots & \cdots & \cdots \\
\cdots & \cdots & \cdots & \cdots & \cdots \\
m_{12} & 0 & \cdots & m_{21} & m_{N N}
\end{array}\right] \\
K= & {\left[\begin{array}{ccccc}
k_{11} & k_{12} & 0 & \cdots & k_{21} \\
k_{21} & k_{22} & k_{12} & 0 & \cdots \\
\cdots & \cdots & \cdots & \cdots & \cdots \\
\cdots & \cdots & \cdots & \cdots & \cdots \\
k_{12} & 0 & \cdots & k_{21} & k_{N N}
\end{array}\right] }
\end{aligned}
$$

where $F$ is the vector of applied forces, $m_{i i}$ and $k_{i i}$ are the blade/sector mass and stiffnesses and $N$ is the number of blades/sectors. $m_{i i}$ and $k_{i i}$ are equal for tuned bladed disks but differ for mistuned bladed disks. 
The EOM inputs $(F)$ and outputs $(y)$ can be any linear combination of blade input forces $(u)$ or responses $(x)$ respectively. For the response of any particular blade $i, C$ is a sparse row vector with $C_{i}=1$.

The forcing function $(F)$ can be written as the product of an input shape matrix $(B)$, which characterizes the spatial properties of the applied forces, and a vector $(u)$, which characterizes the time properties of the forces. Engine order excitation is assumed to be harmonic excitation which differs from blade to blade by a constant interblade phase angle $(\theta)$ :

$$
\begin{aligned}
B_{i} & =e^{j(i-1) \theta} \quad i=1,2, \ldots, N \\
u & =e^{j \omega t} \\
\theta & =2 \pi \frac{E}{N}
\end{aligned}
$$

where $E$ is the engine order.

The solution to Eq. (1) can be written as the summation of modal responses in physical coordinates (The mode shapes are mass normalized so that $\phi^{T} M \phi=I$ ):

$$
\begin{aligned}
y_{i}= & \left(\frac{\phi_{i 1} \phi_{1}^{T} B}{\left(p_{1}^{2}-\omega^{2}\right)}+\frac{\phi_{i 2} \phi_{2}^{T} B}{\left(p_{2}^{2}-\omega^{2}\right)}+\cdots\right. \\
& \left.+\frac{\phi_{i N} \phi_{N}^{T} B}{\left(p_{N}^{2}-\omega^{2}\right)}\right) u
\end{aligned}
$$

where $p$ is a system pole or natural frequency. The frequency response, $h$, is defined by $y=h u$ so that:

$$
\begin{aligned}
h_{i}= & \frac{\phi_{i 1} \phi_{1}^{T} B}{\left(p_{1}^{2}-\omega^{2}\right)}+\frac{\phi_{i 2} \phi_{2}^{T} B}{\left(p_{2}^{2}-\omega^{2}\right)}+\cdots \\
& +\frac{\phi_{i N} \phi_{N}^{T} B}{\left(p_{N}^{2}-\omega^{2}\right)}
\end{aligned}
$$

The frequency response can also be written as the ratio of two factored polynomials in $w^{2}$ :

$$
\begin{aligned}
h_{i}= & \\
& \frac{G\left(z_{1}^{2}-w^{2}\right)\left(z_{2}^{2}-w^{2}\right) \cdots\left(z_{N-1}^{2}-w^{2}\right)}{\left(p_{1}^{2}-w^{2}\right)\left(p_{2}^{2}-w^{2}\right) \cdots\left(p_{N}^{2}-w^{2}\right)}
\end{aligned}
$$

where $z$ is a transfer function zero and $G$ is a constant determined by the static deflection of the structure:

$$
G=K^{-1} B \frac{\left(p_{1}^{2}\right)\left(p_{2}^{2}\right) \cdots\left(p_{N}^{2}\right)}{\left(z_{1}^{2}\right)\left(z_{2}^{2}\right) \cdots\left(z_{N-1}^{2}\right)}
$$

In this paper, Eq. (9) is referred to the modal summation approach, and Eq. (10) is referred to as the transfer function approach. The zeros of Eq. (10) are often referred to as antiresonant frequencies in the literature, because they cause sharp inverted resonant peaks in the frequency response when plotted on a logarithmic magnitude axis (see Fig. 1). To emphasize FRF antiresonances, this paper defines the frequency response function (FRF) to be the magnitude of the frequency response in decibels:

$$
F R F=20 \log |h|
$$

Damping was added to the transfer function FRFs to prevent infinite resonant peaks and antiresonant valleys. Structural damping was incorporated by adding an imaginary part to the undamped poles $\left(p_{u}\right)$ :

$$
p^{2}=p_{u}^{2}+2 \zeta p_{u}^{2} j
$$

Damping was assumed to have a similar effect on the undamped zeros $\left(z_{u}\right)$ :

$$
z^{2}=z_{u}^{2}+2 \zeta z_{u}^{2} j
$$

To validate this approach, the transfer function FRFs were compared to the modal summation FRFs in equation (9) using the classic assumption of constant modal damping where:

$$
p^{2}=p_{u}^{2}+2 \zeta p_{u} \omega j
$$

A $\zeta$ of 0.001 was used for this comparison as well as the numerical examples in this paper. The transfer function and modal summation FRFs were virtually identical.

\subsection{Calculation of transfer function zeros}

Wang [14] showed that the undamped transfer function zeros $\left(z_{u}\right)$ are the eigenvalues of reduced mass and stiffness matrices:

$$
\operatorname{det}\left(\tilde{K}-z_{u}^{2} \tilde{M}\right)=0
$$

where

$$
\begin{aligned}
\tilde{M} & =B_{N}^{T} M C_{N} \\
\tilde{K} & =B_{N}^{T} K C_{N} \\
B_{N} & =\operatorname{nullspace}(B) \\
C_{N} & =\operatorname{nullspace}(C)
\end{aligned}
$$

$\tilde{M}$ and $\tilde{K}$ are non-symmetric matrices whose eigenvalues $\left(z_{i}^{2}\right)$ can be positive, negative, real or complex numbers.

Two observations can be made about the role of zeros in bladed disk FRFs based on the above development. The first observation comes from examining the response of an individual blade such as blade 1 . The $C$ row vector for blade 1 is:

$$
C=\left[\begin{array}{llll}
1 & 0 & 0 & \cdots
\end{array}\right]
$$


The nullspace of $C$ is then:

$$
C_{N}=\left[\begin{array}{c}
0_{1 \times \mathrm{N}-1} \\
I_{\mathrm{N}-1 \mathrm{xN}-1}
\end{array}\right]
$$

where $0_{1 \times \mathrm{N}-1}$ indicates a row of zeros. The choice of blade 1 resulted in the first row of $C_{N}$ containing all zeros. However, any blade $i$ could have been chosen for this development and would have resulted in the $i$ th row of $C_{N}$ containing all zeros. In the postmultiplication of $M$ and $K$ by $C_{N}$ in Eqs (17) and (18), the $i$ th row of $C_{N}$ deletes the $i$ th column from $M$ and $K$. Without the $i^{\text {th }}$ column, $\tilde{M}$ and $\tilde{K}$ do not contain the sector mass and stiffness $m_{i i}$ and $k_{i i}$. Since the zeros are the eigenvalues of $\tilde{M}$ and $\tilde{K}$, they must be independent of $m_{i i}$ and $k_{i i}$. Therefore:

Conclusion 1 The zeros of a blade's FRF are independent of any changes to its own mass and stiffness.

A second observation results from examining engine orders $N$ and $N / 2$. These engine orders cause $B, \tilde{M}$ and $\tilde{K}$ to be real by Eqs (5), (17) and (18). Since the eigenvalues of a real matrix must be real or complex conjugates [15], all zeros are guaranteed to be real or complex conjugates when the engine order is $N$ or $N / 2$. This fact has implications on maximum response, as explained in the next section.

\subsection{Minimizing maximum response}

It is well known that different mistuning patterns can result in very different maximum response amplitudes. Under the modal summation approach, Eq. (9), the mode shapes determine maximum response. The modal participation factors, $\phi_{i n} \phi_{n}^{T} B$, determine whether the modes in the summation respond in phase or out of phase. In phase modes reinforce each other and increase maximum response; out of phase modes do the opposite. But what mode shapes are desirable for minimizing maximum response levels? This is a difficult question, because of the disordered nature of mistuned bladed disk mode shapes [3].

The transfer function formulation has the advantage of providing an intuitive relationship between transfer function zeros and maximum response. Transfer function zeros attenuate forced response just as the poles amplify the forced response. The effect of zero spacing on forced response can be seen in the following example where three FRFs with identical poles but different sets of zeros are shown in Fig. 1. The poles and zeros are listed in Table 1. The first FRF is analogous to a tuned bladed disk FRF, where only one resonant peak is visible [4] (The other resonances are cancelled by the $N-1$ antiresonances). FRFs 2 and 3 represent blade FRFs from two different mistuned rotors that do not contain collocated poles and zeros.

Figure 1 shows that zero spacing is a critical factor in determining maximum response. In FRF 1, the maximum response is determined by the full contribution of one pole to the response without any attenuation from a zero (The zeros are completely cancelled by collocated poles). In FRF 2, the zeros interlace the poles, allowing all poles to be present in the response. However, each pole is partially attenuated by a nearby zero, so that the maximum response is reduced below the "tuned" (FRF 1's) maximum response. In FRF 3, two neighboring poles are left without any interlacing or cancelling zeros between them. The result is that the contribution from the two poles combine to increase the maximum response above the "tuned" case, which only had one pole present in the response. Clearly, for a given set of poles, the spacing of zeros in the FRF determine the maximum response and interlacing poles and zeros are desirable for reducing maximum response below the tuned maximum response.

The other factor that determines maximum response is the complexity of the zeros. More complex zeros appear as increasingly "damped" antiresonances; i.e. they provide less attenuation in the FRF, increasing maximum response. Therefore, the maximum response is minimized when real zeros interlace the system poles. Based on Fig. 1 and the previous discussion, the following conclusion can be given:

Conclusion 2 Mistuning maximum response amplification is caused by damping and inefficient spacing (not interlacing the poles) of antiresonances in the blade FRFs.

Some physical interpretation of why resonant response is reduced by a nearby antiresonance can be gained by examining the modal FRF formulation in Eq. (9). When the excitation frequency equals a natural frequency, the blade response is dominated by a single system mode. However, the residual response contribution from a neighboring mode is not negligible for a system with sufficiently closely spaced natural frequencies. If the residual response contribution has opposite phase to the resonant mode's response contribution, then the two subtract and reduce the magnitude of the resonant response. If the excitation frequency is moved away from one natural frequency and towards the other, the response from one mode decreases as the other increases. Eventually, the contributions from the 


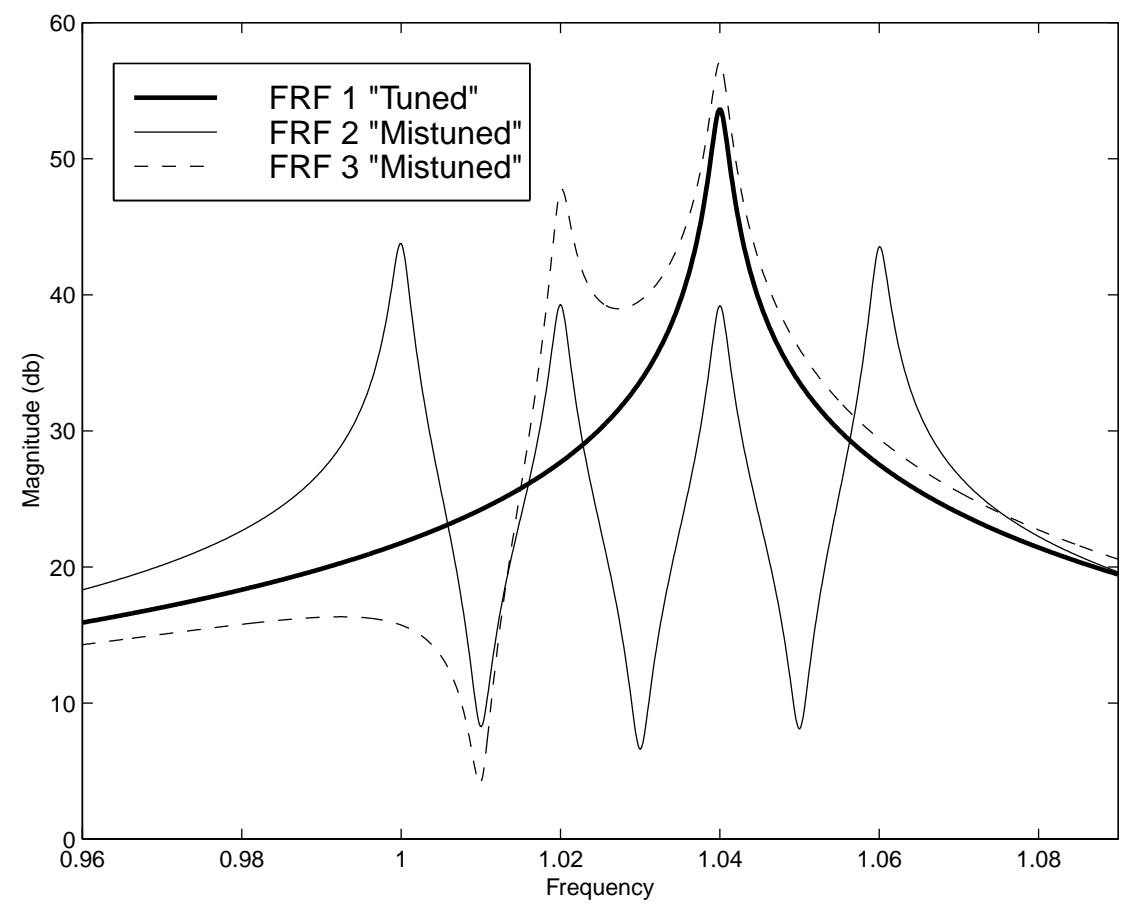

Fig. 1. The effect of different zero spacings on maximum response.

Table 1

FRF Poles and Zeros for Fig. 1

\begin{tabular}{cccc}
\hline Poles & $\begin{array}{c}\text { Zeros for } \\
\text { FRF 1 } \\
\text { "Tuned" }\end{array}$ & $\begin{array}{c}\text { Zeros for } \\
\text { FRF 2 } \\
\text { "Mistuned" }\end{array}$ & $\begin{array}{c}\text { Zeros for } \\
\text { FRF3 } \\
\text { "Mistuned" }\end{array}$ \\
\hline $1.00+0.001 \mathrm{j}$ & $1.00+0.001 \mathrm{j}$ & $1.01+0.001 \mathrm{j}$ & $1.00+0.001 \mathrm{j}$ \\
$1.02+0.001 \mathrm{j}$ & $1.02+0.001 \mathrm{j}$ & $1.03+0.001 \mathrm{j}$ & $1.01+0.001 \mathrm{j}$ \\
$1.04+0.001 \mathrm{j}$ & $1.06+0.001 \mathrm{j}$ & $1.05+0.001 \mathrm{j}$ & $1.06+0.001 \mathrm{j}$ \\
$1.06+0.001 \mathrm{j}$ & & & \\
\hline
\end{tabular}

two modes become equal in magnitude and opposite in phase, creating zero response, which is an antiresonant frequency. Therefore, antiresonant frequencies are indications that the response contributions from neighboring modes are opposite in phase and destructively interfering with each other.

Having established the effect of antiresonance on maximum response, we can now revisit case of engine orders $\mathrm{N}$ and $\mathrm{N} / 2$. Recall from the last section that these engine orders cause all antiresonances to be real or complex conjugate pairs. Neither real or complex conjugate undamped zeros are ideal for minimizing maximum response. Real zeros provide the same attenuation in the FRF as the poles provide amplification, because the system poles are also real and use the same damping assumption as the zeros in Eqs (13) and (14). Ideally, it is desirable for the damped zeros in
Eq. (14) to be less complex than the poles in Eq. (13) so that they provide more attenuation in the FRF.

Complex conjugate zeros are also detrimental to minimizing maximum response. A pair of complex conjugate zeros will occur at the same frequency in the FRF. These "double zeros" leave fewer zeros to attenuate other areas of the FRF. The result is an inefficient spacing of zeros in the FRF (See Conclusion 2). Therefore, engine orders $\mathrm{N}$ and $\mathrm{N} / 2$ will tend to produce higher maximum response than other engine orders.

Conclusion 3 Engine orders $N$ and N/2 tend to produce higher maximum response than other engine orders, because the undamped antiresonances are guaranteed to be either real or complex conjugate pairs.

This conclusion is consistent with the findings of Kenyon et al. [16] who showed that the maximum amplitude magnification due to mistuning is greater for 


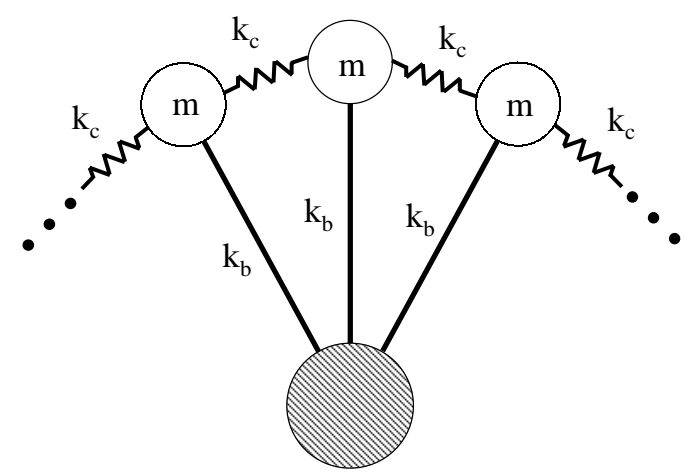

Fig. 2. Coupled oscillator model.

engine orders $\mathrm{N}$ and $\mathrm{N} / 2$ when simple damping is assumed. Similarly, Whitehead [18] concluded that engine orders $\mathrm{N}$ and $\mathrm{N} / 2$ (where $\mathrm{N}$ is even in the case of $\mathrm{N} / 2$ ) guarantees that the maximum amplitude magnification factor can be achieved.

\section{Numerical examples}

\subsection{Bladed disk model}

The coupled spring-mass oscillator model shown in Fig. 2 was used to demonstrate the role of antiresonance in mistuning response amplification. Although mistuning is normally applied in the literature by altering individual blade stiffnesses, in this paper mistuning was accomplished by changing the individual blade masses. The resulting mass and stiffness matrices are given by:

$$
\begin{aligned}
M= & \operatorname{diag}\left(\frac{m_{1}}{m}, \frac{m_{2}}{m}, \cdots, \frac{m_{N}}{m}\right) \\
K= & \omega_{b}^{2} \text { circular } \\
& (1+2 R,-R, 0, \cdots, 0,-R) \\
\omega_{b}^{2}= & \frac{k_{b}}{m}=1 \\
R= & \frac{k_{c}}{k_{b}}=0.01
\end{aligned}
$$

where $\omega_{b}, m$ and $k_{b}$ are the nominal blade natural frequency, mass and stiffness.

The reason for mistuning the blade masses can be seen in Fig. 3(a). Here the mistuning amplification (A) is plotted versus the standard deviation of the mistuning where mistuning amplification is defined as the ratio of maximum mistuned response to maximum tuned response. With stiffness mistuning, the low frequency blade (blade 1 in Fig. 3(b)) approaches zero stiffness at higher mistuning levels. This flexible blade will have high response across all frequencies in its FRF (Eq. (11)), causing mistuning amplification to increase with mistuning standard deviation. Therefore, mass mistuning was used to focus solely on the response amplification that is caused by the breakdown of pole/zero cancellations in Eq. (10).

\subsection{Engine order excitation}

A 12 bladed coupled oscillator model was used to investigate the effect that different engine order excitations have on maximum response. Mistuning patterns were created by randomly selecting blade frequencies from a normal distribution with a mean of 1 and a standard deviation of $2 \%$. The forced response of 50,000 random mistuning patterns were solved for each engine order excitation. The probability density functions (pdf) of the resulting mistuning amplifications are shown in Fig. 4.

As predicted by Conclusion 3, engine orders 6 and 12 produced the highest average mistuning amplification, which was approximately $50 \%$ greater than the tuned response. Interestingly, some mistuned rotors had lower maximum response than the tuned rotor $(A<1)$. This phenomena, although present in papers by Turcotte [17] and Castanier [1], has received little attention in the literature. The cause of this mistuning attenuation is investigated in section 3.3. Another interesting note is that engine orders $1,5,6$, and 12 seemed incapable of producing less than the tuned maximum response.

The role of antiresonance in determining maximum response was demonstrated by comparing pole/zero maps in the complex plane with magnitude FRFs. An example of this method is shown in Fig. 5. The real axis of the pole/zero map correlates with the FRF frequency axis. The tuned FRF is plotted in bold to emphasize the bandwidth of a pole (or zero with $\operatorname{Im}(z)=0.001 j$ ) and the tuned maximum response. Note that the system poles always lie at 0.001 on the imaginary axis, because of the damping assumption in Eq. (13). Zeros that lie between the two dashed lines at $\pm 0.001 j$ are more lightly damped than the poles. Therefore, they reduce maximum response by providing more attenuation in the FRF than the poles provide amplification.

The maximum response of a mistuned bladed disk subjected to engine order $\mathrm{N}$ was examined in further detail in Fig. 5. The pole/zero map of the maximum 

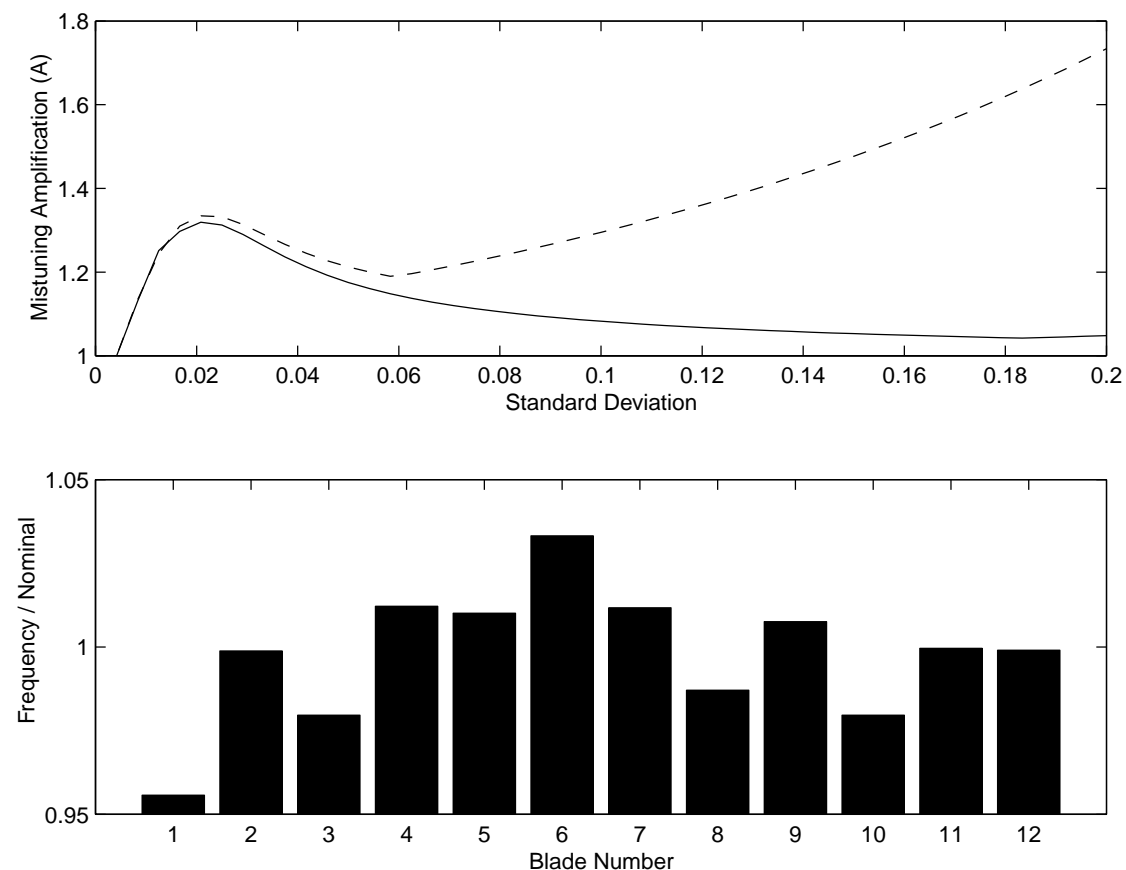

Fig. 3. a) Comparison of mass and stiffness mistuning $(E=2)$. - mass mistuning, - - - stiffness mistuning. b) Mistuning pattern.

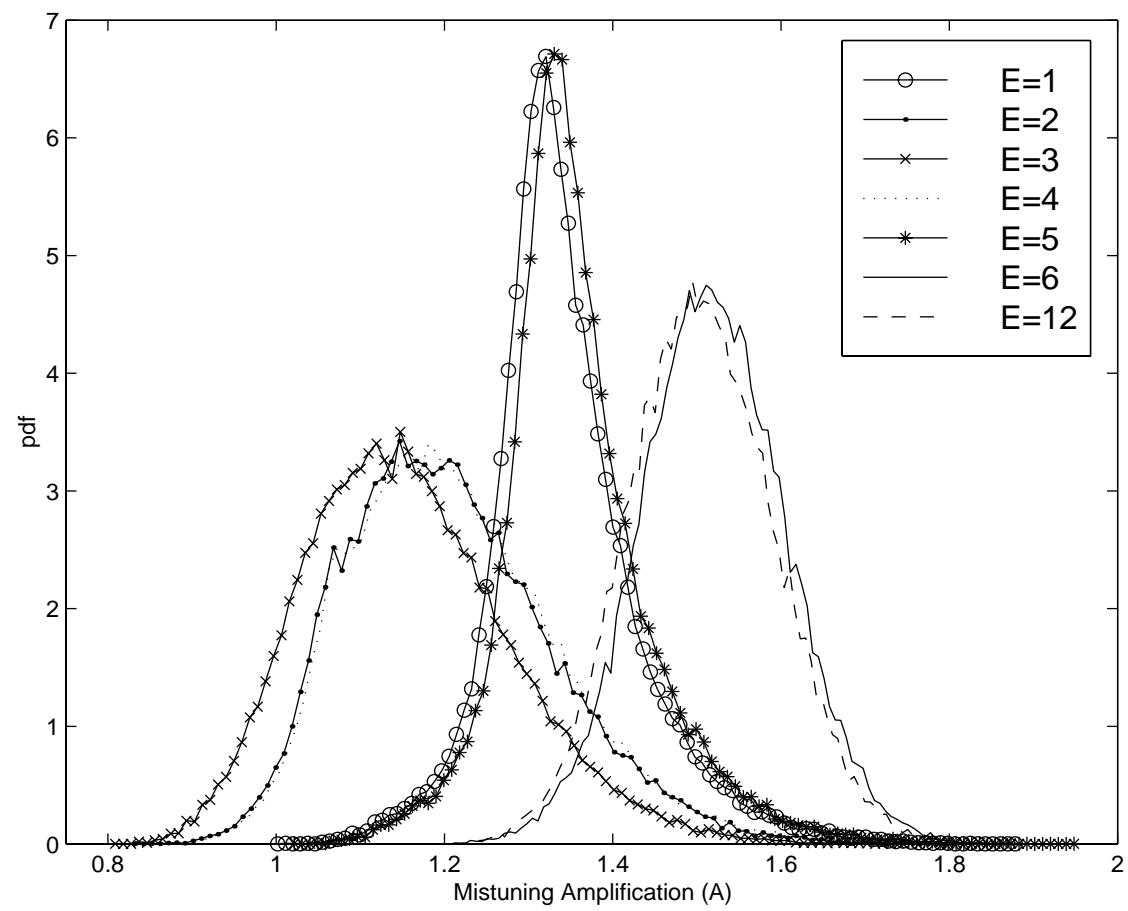

Fig. 4. Probability density functions of mistuning amplification for various engine orders.

responding blade FRF contains three sets of complex conjugate zeros, symmetric about $0.001 j$. These three sets of zeros create antiresonances in the FRF at the same frequency. The middle complex conjugate zero pair (near 1.01 on the real axis) causes a sharp antiresonance because of one lightly damped zero between 

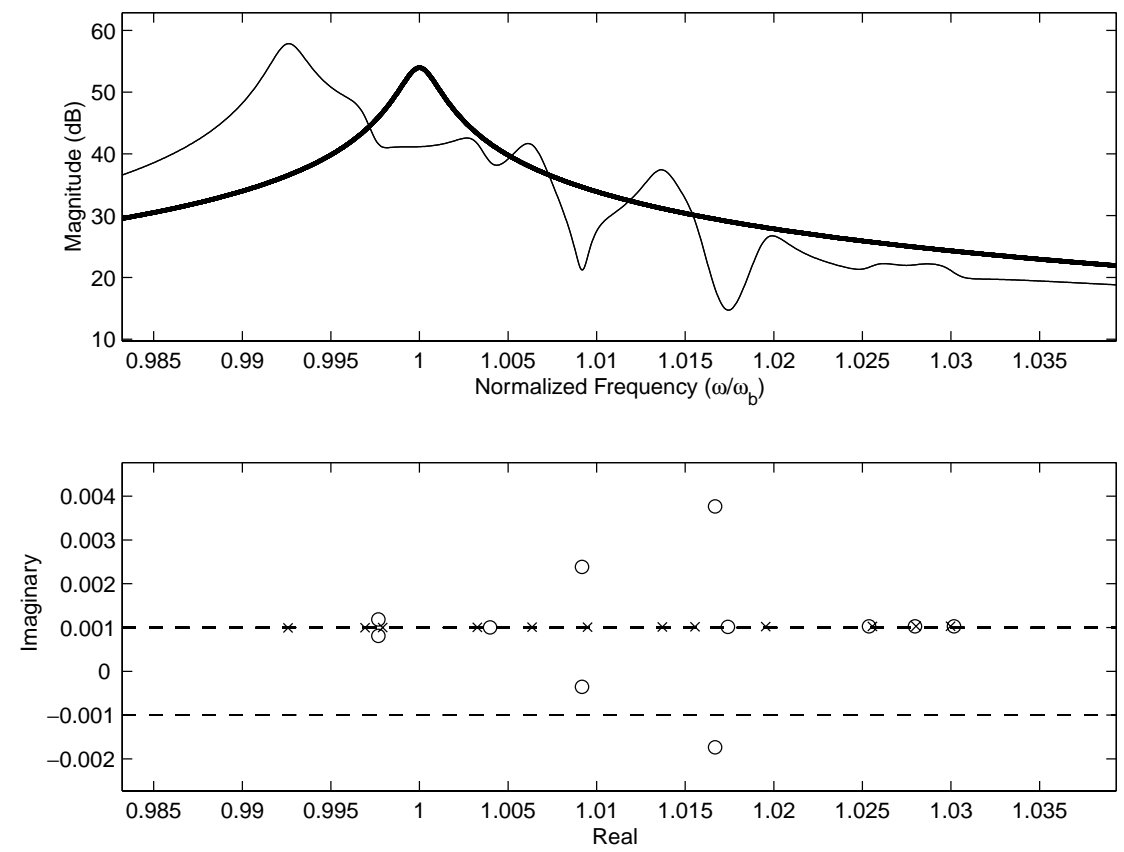

Fig. 5. Response to Engine Order N. a) maximum Blade FRF $(A=1.56)$. — tuned, - mistuned. b) Pole/Zero Map. $\times$ poles, $\circ$ zeros. Zeros between the dashed lines reduce the response more than the poles amplify it.

the dashed lines. All but two zeros lie on or outside of the dashed lines, indicating that they are more damped than tuned zeros. The complex conjugate zeros "use up" two many zeros at the high frequency side of the FRF, not leaving enough zeros to cover poles at the low frequencies. Two poles are left at the low frequency end of the FRF with no interlacing zero. As predicted by Conclusion 2, the damping and inefficient spacing of antiresonances increase maximum response over the tuned FRF.

Figure 5 was found to be typical of maximum response FRFs for mistuned bladed disks in that the average zero was more complex (damped) than the poles. This leads to a simple explanation for why mistuning amplification increases with the number of blades [18, 4]. As the number of blades increases, each new blade adds a resonance and antiresonance to the narrow frequency band in the FRF. Because the antiresonance is usually more damped than the resonance, there is a new contribution to the maximum response from this pair.

\subsection{Best and worst case mistuning patterns}

The largest and smallest mistuning amplifications for engine order 3 were identified from the 50,000 solutions used to create the pdf in Fig. 4. These mistuning patterns were used to investigate the extremes of antiresonance behavior in bladed disk FRFs. Engine order 3 was chosen because it produced the greatest reduction in maximum response from the tuned bladed disk.

Figure 6 shows the FRF and pole/zero map of the maximum responding blade in the worst case mistuning pattern. The peak responding blade has no lightly damped antiresonances appearing in the FRF. The pole/zero map shows that the antiresonances are more complex (damped) than the poles. Finally, there are two poles with no interlacing zeros in the highest response region of the FRF. The result is an $84 \%$ increase in maximum response over a tuned bladed disk.

Figure 7 shows the FRF of the best case mistuning pattern. In this case, even the peak responding blade FRF has three very lightly damped antiresonances in the response. Furthermore, the antiresonances are spread evenly across the FRF so that they either interlace or very nearly cancel the poles. These two factors combined to produce a $18 \%$ reduction over tuned response and a $34 \%$ reduction over the mean mistuned maximum response from the distribution in Fig. 4. If this mistuning state could be maintained in a turbine engine, it would significantly extend bladed disk life.

\subsection{Antiresonance sensitivity to mistuning}

Wei and Pierre [3] showed that bladed disk eigenvalues $\left(p_{u}^{2}\right)$ and eigenvectors $(\phi)$ are very sensitive to 

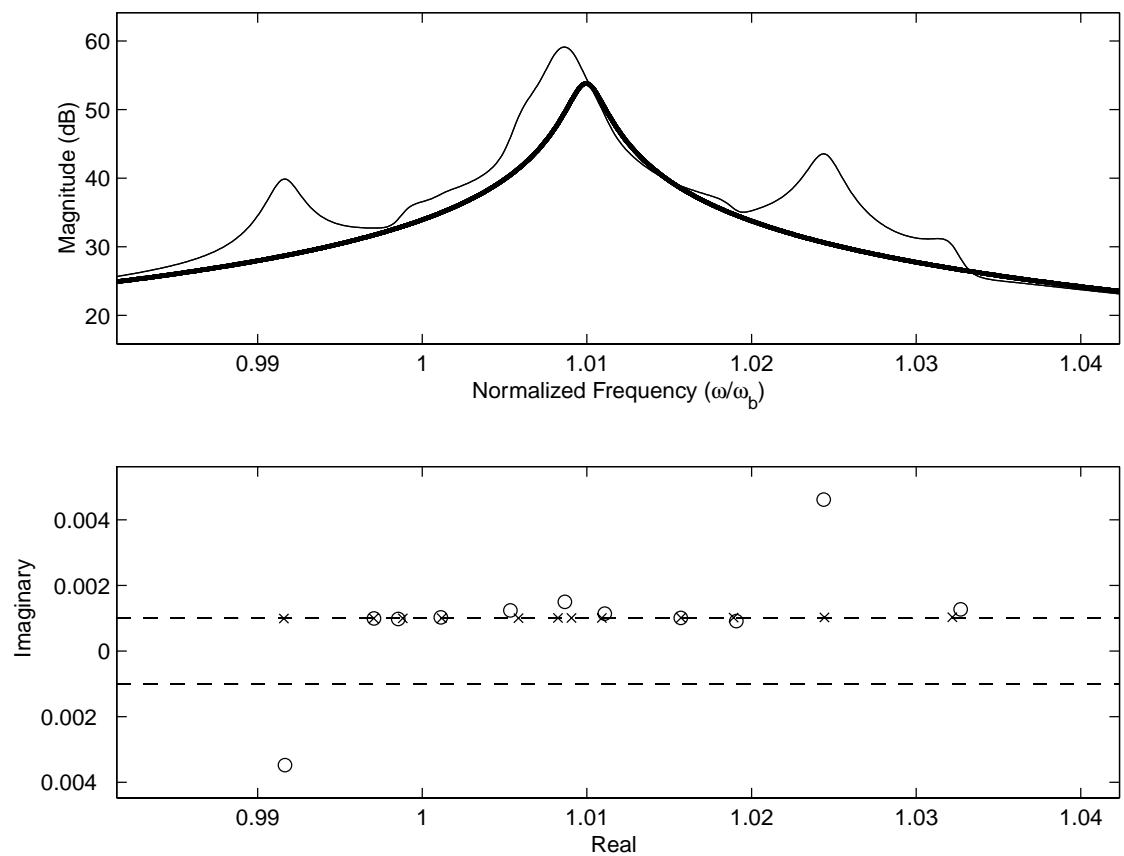

Fig. 6. Largest Amplification Mistuning Pattern ( $\mathrm{A}=1.84, \mathrm{E}=3)$. a) Maximum Blade FRF. — tuned, - mistuned. b) Pole/Zero Map. $\times$ poles, $\circ$ zeros. Zeros between the dashed lines reduce the response more than the poles amplify it.
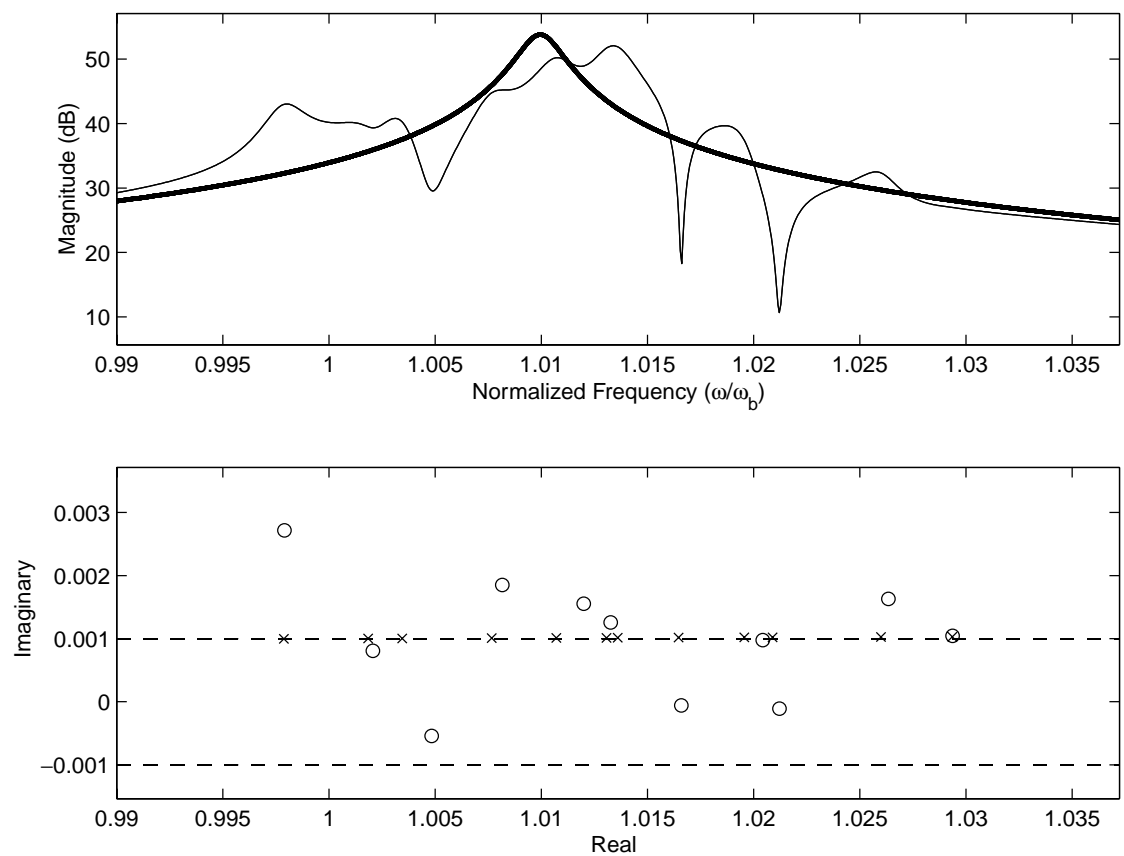

Fig. 7. Smallest Amplification Mistuning Pattern $(A=0.82, E=3)$. a) Maximum Blade FRF. — tuned, - mistuned. b) Pole/Zero Map. $\times$ poles, $\circ$ zeros. Zeros between the dashed lines reduce the response more than the poles amplify it.

the amount of mistuning. The closely spaced eigenvalues of the tuned system "veer" away from each other as the amount of mistuning is increased. The tuned eigenvectors change from having extended participation in many blades to being more localized around one or a few blades when mistuning is increased. Since 


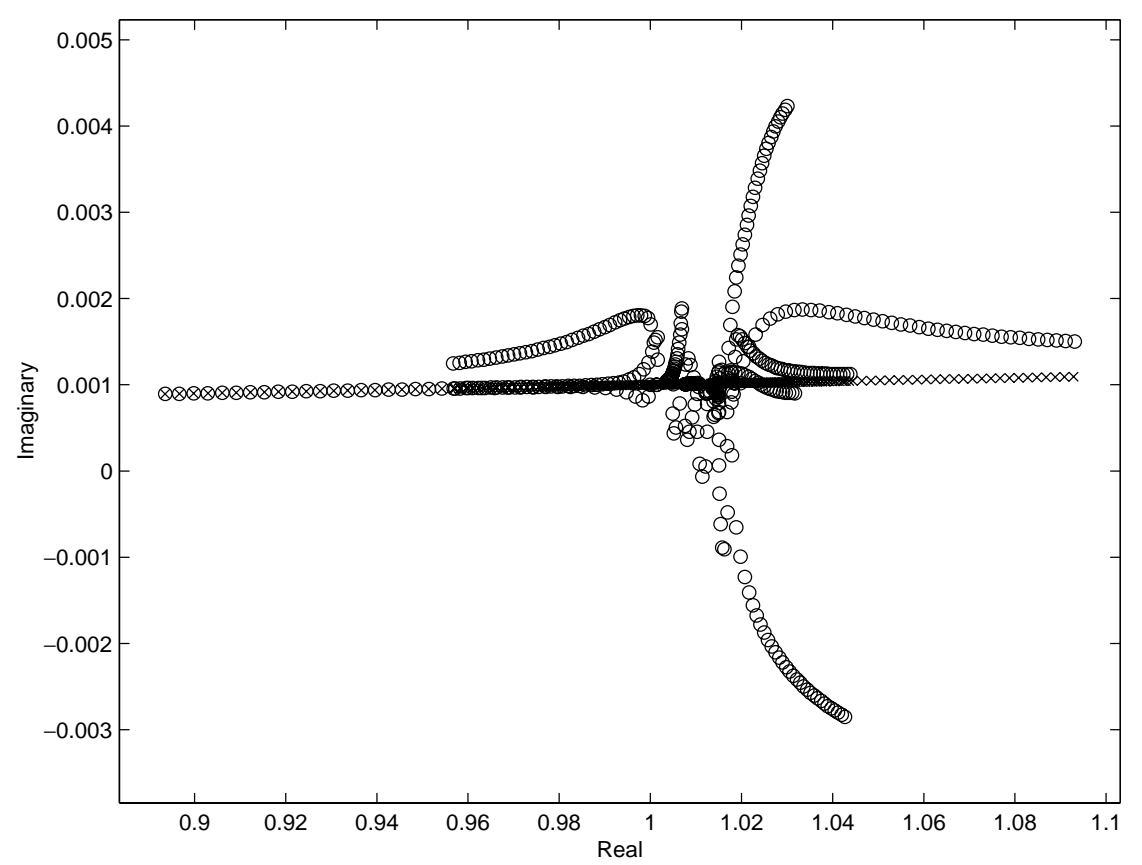

Fig. 8. Blade 8 pole/zero locus as mistuning standard deviation is varied from 0 to 0.1 for the mistuning pattern given in Fig. $3($ b). $\times$ poles, ○ zeros.

bladed disk antiresonances are defined by the system eigenvalues and eigenvectors (i.e. they are the zeros of Eq. (9)), they show a similar sensitivity to the amount of mistuning.

Consider the mass mistuning example from Fig. 3 . Since there is a different set of antiresonances for each blade's FRF, a representative blade must be chosen for examination. Blade 8 was chosen because it was the maximum responding blade between a standard deviation of 0.01 and 0.1 in Fig. 3a. Blade 8's pole/zero locus as mistuning standard deviation is increased is shown in Fig. 8. Figure 8 shows that the zeros spread along the real axis along with the veering poles. The complexity of the zeros varies wildly with small mistuning, with more zeros increasing their complexity than decreasing. As the mistuning gets larger, all but two antiresonances are returning to the same complexity as the poles. The temporary increase in zero complexity corresponds with the high mistuning amplification seen between 0.01 and 0.05 in Fig. 3a. This supports the conclusion that mistuning amplification is related to the damping (increased complexity) of the antiresonances.

Lastly, to validate Conclusion 1 , the sensitivity of a blade's antiresonances to changes to its own mass was investigated. Figure 9 shows the effect on a blade's FRF when mistuning is applied to that blade only. The natural frequency of the blade whose FRF is shown in Fig. 7 was increased by $1 \%$ of the nominal blade frequency $\left(\omega_{b}\right)$. This blade's FRF and pole/zero map are shown before and after the self-mistuning in Fig. 9. As predicted by Conclusion 1, the zeros are insensitive to the mistuning. However, the FRF changes due to the movement of the poles. As a further note, the blade is no longer the peak responding blade after self-mistuning.

\section{Conclusions}

This paper applied antiresonance theory to the forced response of bladed disks to engine order excitation. Bladed disk frequency response was formulated using a transfer function approach. Using this method, the response can be written completely in terms of eigenvalues and antiresonances without using mode shapes. Since antiresonances create attenuation in the FRF, their effect on maximum response can be easily understood.

The transfer function formulation led to the following conclusions:

- The zeros of a blade's FRF are independent of any changes to its own mass and stiffness.

- Mistuning maximum response amplification is caused by damping and inefficient spacing of antiresonances in the blade FRFs. 

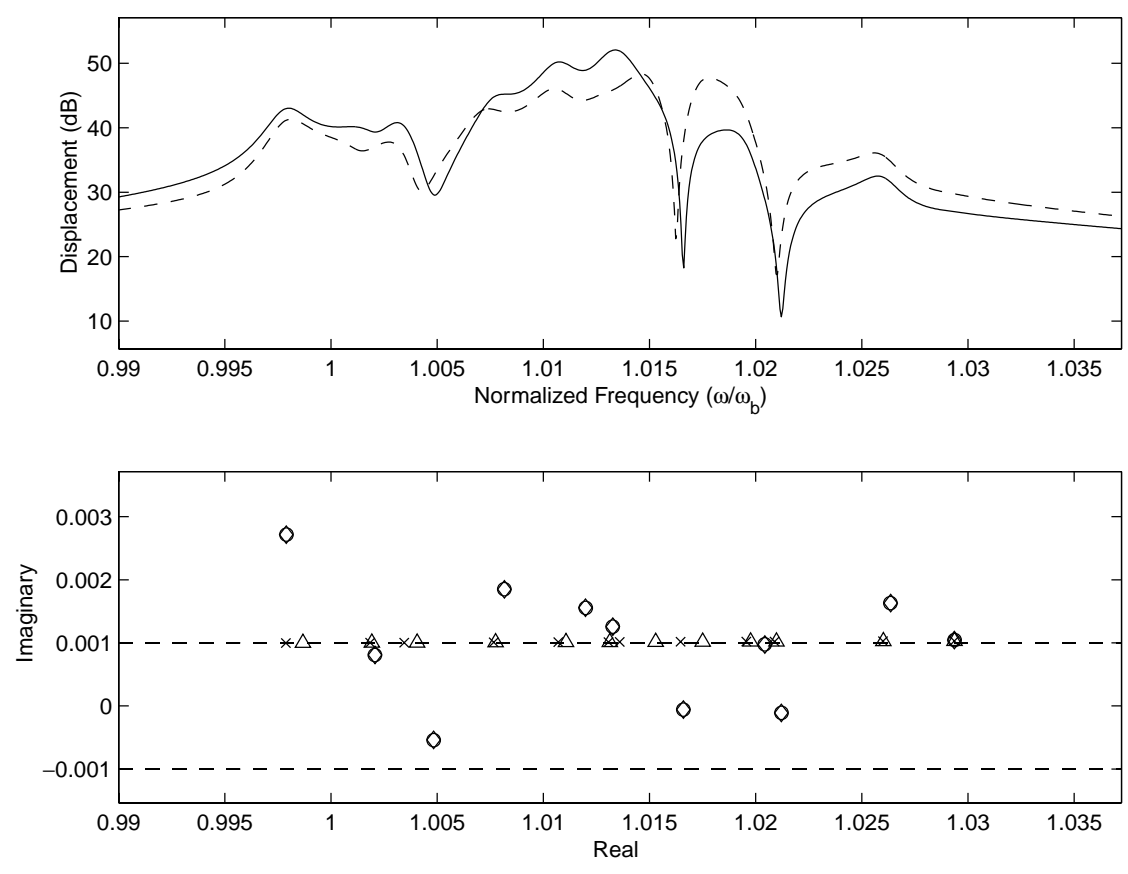

Fig. 9. Blade FRF Sensitivity to Self Mistuning (E=3). a) Blade FRF. - Before, - - After. b) Pole/Zero Map. ( $\times$,o) - (pole, zero) before, $(\triangle, \diamond)$ - (pole, zero) after. Zeros between the dashed lines reduce the response more than the poles amplify it.

- Engine orders $\mathrm{N}$ and N/2 tend to produce higher maximum response levels than other engine orders, because the undamped antiresonances are guaranteed to be either real or complex conjugate pairs.

- Mistuning amplification increases with the number of blades, because the antiresonances are usually more damped than the closely spaced resonances.

- Mistuned bladed disks can have lower maximum response than tuned bladed disks. This mistuning attenuation is caused by lightly damped antiresonances that are spread throughout all blade FRFs.

Robust minimum maximum response mistuning designs for bladed disks would be an attractive weapon against high cycle fatigue. Since antiresonance sensitivities can be easily calculated [14], structural optimization routines may be able to find such mistuning patterns using the reduction of the imaginary part of the antiresonances as a goal. This is a recommended area for further research.

\section{References}

[1] M.P. Castanier and C. Pierre, Consideration of the Benefits of Intentional Mistuning for the Forced Response of Turbomachinery Rotors, Proceedings of the 1997 International Mechanical Engineering Congress and Exposition, Dallas, TX, USA, 1997.
[2] D.J. Ewins, The Effects of Detuning Upon the Forced Vibrations of Bladed Disks, Journal of Sound and Vibration 9(1) (1969), 65-79.

[3] S. Wei and C. Pierre, Localization Phenomena in Mistuned Assemblies with Cyclic Symmetry Part I: Free Vibrations, Journal of Vibration, Acoustics, Stress, and Reliability in Design 110 (Oct 1988), 429-438.

[4] S. Wei and C. Pierre, Localization Phenomena in Mistuned Assemblies with Cyclic Symmetry Part II: Forced Vibrations, Journal of Vibration, Acoustics, Stress, and Reliability in Design 110 (Oct 1988), 439-449.

[5] M.E. Brewer, M.P. Castanier and C. Pierre, Effects of Harmonic Intentional Mistuning on the Free Response of Bladed Disks, Proceedings of the 1999 ASME Design Engineering Technical Conferences, Las Vegas, Nevada, USA, Sep 1999.

[6] J.C. Slater, G.R. Minkiewicz and A.J. Blair, Forced Response of Bladed Disk Assemblies - A Survey, The Shock and Vibration Digest: Center for Intelligent Materials Systems and Structures 31(1) (Jan 1999), 17-24.

[7] D.K. Miu, Physical Interpretation of Transfer Function Zeros for Simple Control Systems with Mechanical Flexibilities, Journal of Dynamic Systems, Measurement, and Control 113 (Sep 1991), 419-424.

[8] M. La Civita and A. Sestieri, On Antiresonance Interpretation and Energy Concentration Along Continuous OneDimensional Systems, Proceedings of the 15th International Modal Analysis Conference, 1997, pp. 778-784.

[9] F. Wahl and L. Forrai, On the Significance of Antiresonance Frequencies in Experimental Structural Analysis, Journal of Sound and Vibration 219(3) (1999), 379-394.

[10] K. Jones and J. Turcotte, Finite Element Model Updating Using Antiresonant Frequencies, Journal of Sound and Vibration 252(4) (May 2002), 717-727. 
[11] W. D'Ambrogio and A. Fregolent, The Use of Antiresonances for Robust Model Updating," Journal of Sound and Vibration 236 (Sep 2000), 227-243

[12] D.A. Rade, G. Lallement and L.A. da Silva, A Strategy for the Enrichment of Experimental Data as Applied to an Inverse Eigensensitivity-Based F.E. Model Updating Method, Proceedings of the 14th International Modal Analysis Conference, 1996, pp. 1078-1085.

[13] G. Lallement and S. Cogan, Reconciliation Between Measured and Calculated Dynamic Behaviors : Enlargement of the Knowledge Space, Proceedings of the 10th International Modal Analysis Conference, 1992, pp. 487-493.

[14] B.P. Wang, Antiresonance and its Sensitivity Analysis in Structural Systems, AIAA Paper 98-1751, 1998, pp. 431-440.

[15] D.G. Zill and M.R. Cullen, Advanced Engineering Mathemat- ics, PWS-Kent Publishing, Boston, 1992, pp. 435.

[16] J.A. Kenyon, J.H. Griffin and D.M. Feiner, Maximum Bladed Disk Forced Response from Distortion of a Structural Mode, ASME Paper GT-2002-30426 International Gas Turbine Institute ASME Turbo Expo 2002, Amsterdam, the Netherlands, 2002.

[17] J.S. Turcotte, A Coupled Plate Model of a Bladed Disk Assembly, Proceedings of the 35th AIAA/ASME/SAE/ASEE Joint Propulsion Conference, Los Angeles, California, USA, Jun 1999.

[18] D.S. Whitehead, The Maximum Factor by Which Forced Vibration of Blades Can Increase Due to Mistuning, Journal of Engineering for Gas Turbines and Power 120 (Jan 1998), 115-119. 

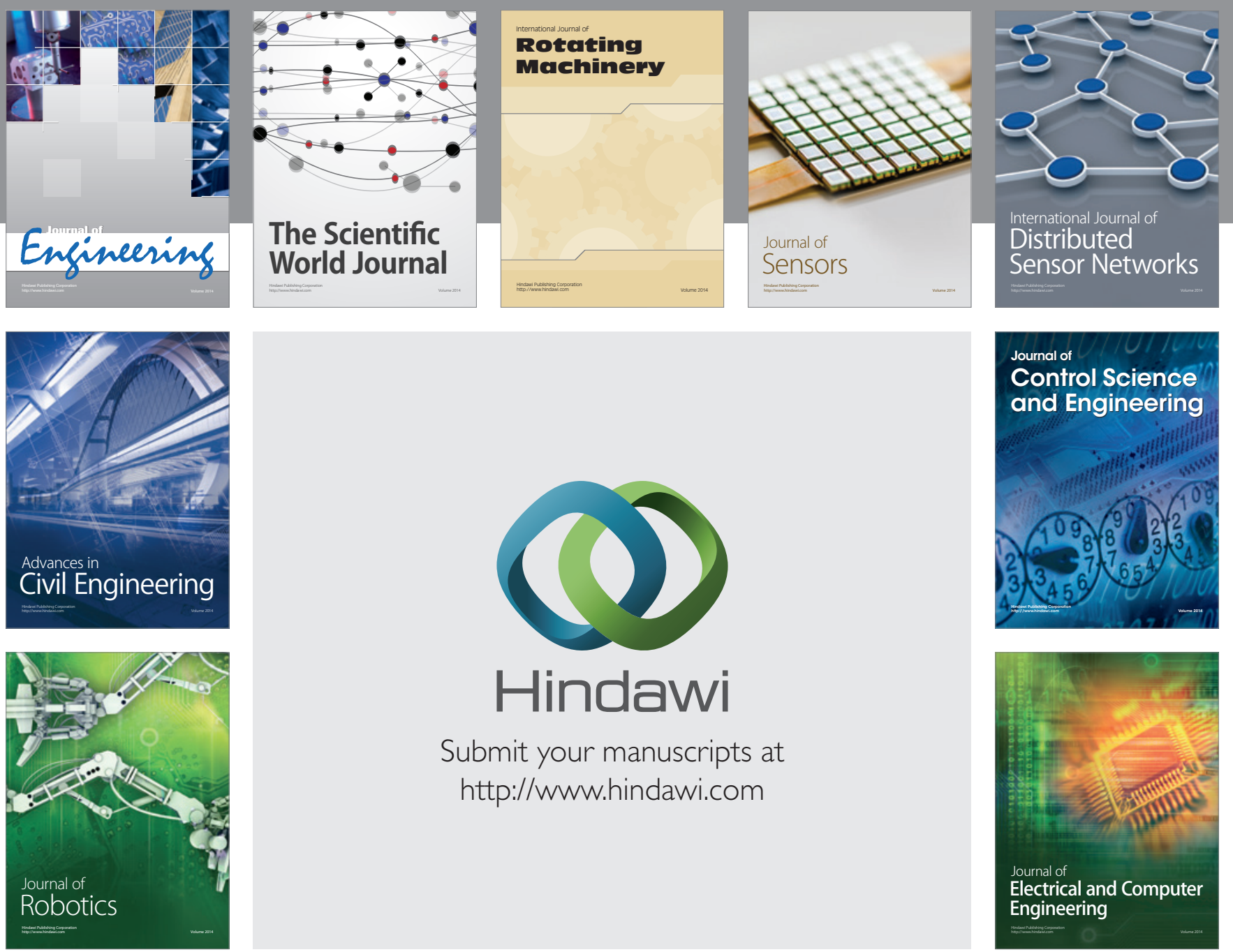

Submit your manuscripts at

http://www.hindawi.com
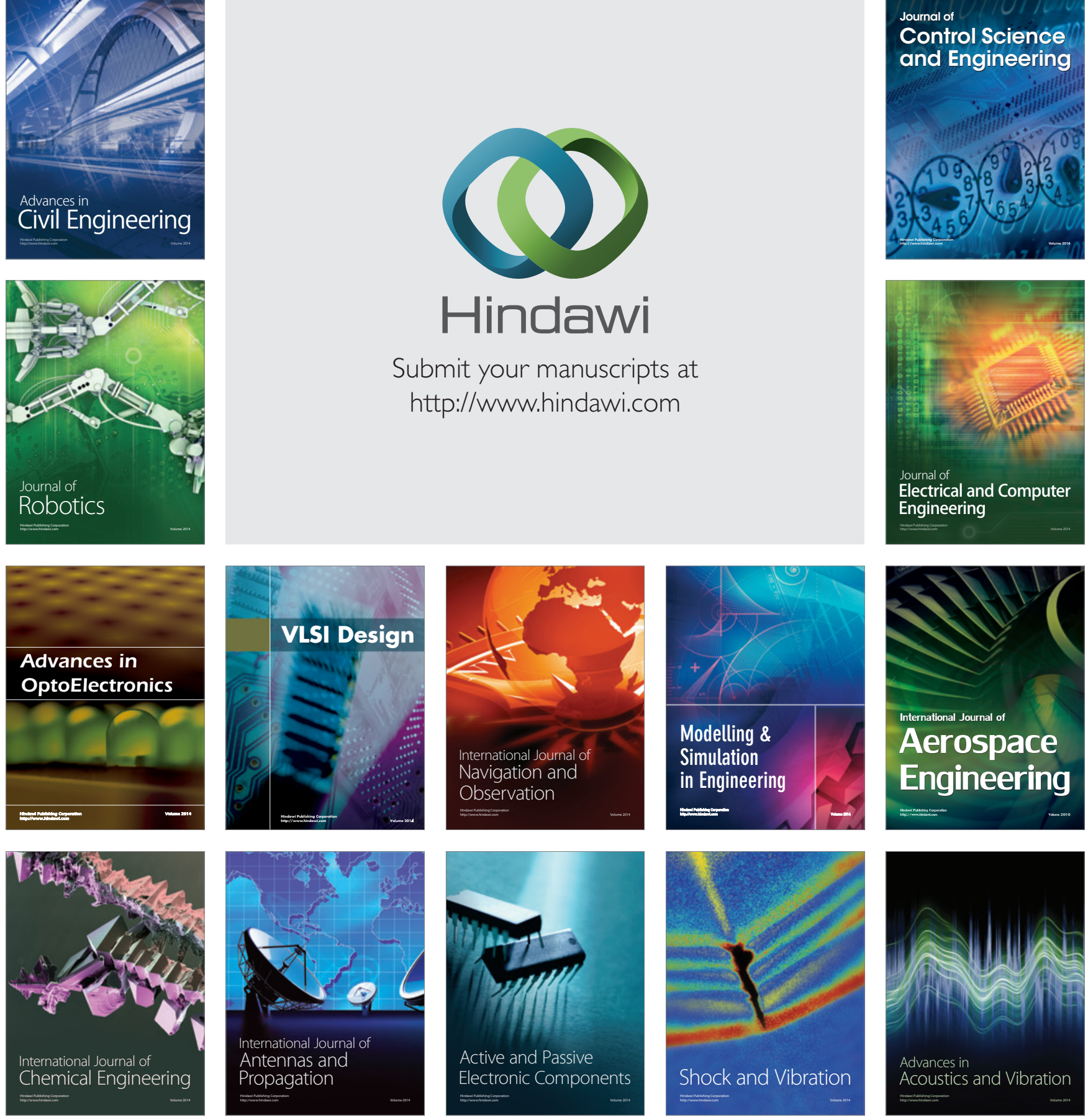